\title{
THE ASYMPTOTIC BEHAVIOUR DUE TO A PIECEWISE TIME DEPENDENT NET MATERNITY FUNCTION
}

\author{
P. CERONE and K. P. TOGNETTI
}

(Received 4 February 1981)

(Revised 26 May 1981)

\begin{abstract}
The long term asymptotic behaviour of a population is evaluated where the age specific fertility behaviour is allowed to change with time. Thus in this article the behaviour of a population is determined with a time dependent net maternity function. It is shown that methods used when the net maternity function was independent of time are still applicable if the change with time is explicit only for the initial population. Further, using the fact that for realistic situations the net maternity function is non-zero over a finite interval $\alpha<x<\beta$, it is shown that traditional methods can again be used if the time dependence is associated with ages less than $\alpha$, the minimum age of childbearing. Recent extensions of Cerone and Keane to include exponential time dependence are utilized and models are presented which are piecewise defined, allowing general and exponential time dependence for the parent and new-born populations respectively. The Sharpe-Lotka single sex determinstic population model is used as the basis for the analysis.
\end{abstract}

\section{Introduction}

A number of authors in the recent past have been concerned with determining the asymptotic effect of changes in the net maternity function in particular through changes in the age specific birth rate. In order to determine the ultimate effect of contraception on a population, Keyfitz [7] abruptly altered the age specific birth rate to replacement level at the origin so that the population would eventually

(C) Copyright Australian Mathematical Society 1982 
level out (stationary). Keyfitz was successful in obtaining an expression for the ultimate population size and thus demonstrating that population growth had a "momentum". He showed that even though the age specific birth rate is abruptly reduced to replacement level, the asymptotic total number in the population is greater than the initial.

Since the pioneer article of Keyfitz [7] a number of generalisations and extensions have appeared in the literature. Frauenthal [4], Tognetti [15] and Mitra [11] presented models which consisted of abrupt changes of the age specific birth rate, while Cerone and Keane [2], [3], gave gradual models. Frauenthal [4] found the asymptotic results due to differential maternity behaviour between the parent and the new-born populations. He analyzed a model also mentioned in Keyfitz [7] which consisted of an abrupt change to replacement level only for the new-born population while the parent population continued with the old regime.

In this article we describe circumstances in which the asymptotic behaviour can readily be obtained using the deterministic single sex population model developed by Sharpe and Lotka [14]. It is shown that the asymptotic behaviour can be determined for a time dependent net maternity function in a manner similar to that used for the time independent models provided the change affects only those individuals alive at the origin. A special case of this is when the change occurs over a period not greater than the minimum age of childbearing. In such a situation, the net maternity function of the new-born population does not change with time. A number of models for this condition are presented and the asymptotic behaviour is determined. Only the asymptotic behaviour of the total birth rate, $B(t)$, is analysed in the present article since that of the total population and age distribution, information which is usually wanted, follow without too much difficulty (see Pollard [12]).

Cerone and Keane [2], [3], developed a fast converging numerical algorithm for determining the asymptotic behaviour of a population when the net maternity function changed exponentially with time. Using these results, the period over which the net maternity function changes with time can be extended. One could postulate that the effects of policies aimed at changing the age-specific birth rate are slow at first, then accelerate only to slow down as a set target is approached. Ruzicka [13] sees the change as being like "an upside down logistic". Frejka [5] and Keyfitz [8] also postulate gradual initial changes.

Using the above ideas as motivation, models of the time dependent net maternity function are presented which are piecewise defined and the asymptotic total birth rate is determined. The model consists of general time dependent changes for the parent population with exponential time dependent variation for the population born after the origin. The methods of Cerone and Keane are utlilized to perform the analysis. 


\section{The basic formulation}

Consider the integral equation describing the total birth rate $B(t)$ for a single sex deterministic population (Keyfitz [6], [9])

$$
B(t)=F(t)+\int_{0}^{t} B(t-x) \phi(x) d x .
$$

Here

$$
\phi(x)=m(x) l(x)
$$

is called the net maternity function with $\phi(x) d x$ representing the fraction of newborn females giving birth to a female child in the age interval $x$ to $x+d x$. The quantity $m(x)$ is the age specific birth rate and $l(x)$ is the survivor function which denotes the fraction of newborn females that will survive to age $x$. It may be seen that $R=\int_{0}^{\infty} \phi(x) d x$ is the net number of births produced during a mother's lifetime and it will be verified that the population will become stationary, with replacement level reproductive behaviour $(R=1)$.

In (1), $F(t)$ represents the contribution to $B(t)$ due to individuals of the parent population and the integral term gives the contribution due to individuals born since the origin.

The solution to (1) is obtained by taking Laplace transforms to yield

$$
B^{*}(p)=F^{*}(p)+B^{*}(p) \phi^{*}(p),
$$

where we have used the notation that the Laplace transform of a function $f(x)$ is $f^{*}(p)=\int_{0}^{\infty} e^{-p x} f(x) d x$. Also in obtaining (2) we have used the convolution theorem which states that

$$
\int_{0}^{\infty} e^{-p t} B(t-x) \phi(x) d x=B^{*}(p) \phi^{*}(p) .
$$

By simple algebra we have, from (2)

$$
B^{*}(p)=F^{*}(p) /\left(1-\phi^{*}(p)\right) .
$$

The inversion of (4) to recover $B(t)$ involves obtaining the contribution from the zeros of

$$
h(p)=1-\phi^{*}(p)
$$

where we are assuming that $F^{*}(p)$ is an entire function (Lopez [10]). For $\phi$ a non-nonegative function it can be established (Bellman and Cooke [1] or Pollard [12]) that there exists a unique real zero $r$ of (5) such that $\operatorname{Re}\left(p_{j}\right)<r$ where $p_{j}$ are the complex zeros. That is, the real zero $r$ has the greatest real part and $r$ is positive, negative or zero according as $R-1$ is positive, negative or zero where

$$
R=\phi^{*}(0)=\int_{0}^{\infty} \phi(x) d x .
$$


Further, the complex zeros of (5) appear in conjugate pairs, which is to be expected since the solution we are seeking is real.

Since the real zero $r$ is greater than the real part of all the complex zeros, we have the asymptotic behaviour given by (Lopez [10], Bellman and Cooke [1])

$$
B(t) \sim \lim _{p \rightarrow r}(p-r) e^{p t} B^{*}(p)=Q e^{r t},
$$

where, from (4) and (5),

$$
Q=F^{*}(r) / \kappa,
$$

and

$$
\kappa=-\left[\frac{d}{d p} \phi^{*}(p)\right]_{p=r}=\int_{0}^{\infty} e^{-r x} x \phi(x) d x .
$$

Here, $\kappa$ is the expected age of giving birth in the stable population.

It should be noted that $r$ (the real zero of (5)) is dependent upon the net maternity function and $Q$, through (7), is a function of both the initial conditions and of $r$.

\section{The time dependent net maternity function}

The integral equation corresponding to (1) for the total birth rate when the net maternity function $\Phi(x, t)$ varies with time $t$ and age $x$ is given by

$$
B(t)=F(t)+\int_{0}^{t} B(t-x) \Phi(x, t) d x,
$$

where

$$
F(t)=\int_{t}^{\infty} B(t-x) \Phi(x, t) d x=\int_{0}^{\infty} B(-x) \Phi(x+t, t) d t
$$

and

$$
B(-x)=N(0) a(x, 0) / l(x) .
$$

Here, (see for example Keyfitz [6]),

$N(0)$ is the initial number in the population,

$a(x, 0)$ is the initial age density function, and

$l(x)$ is the survivor function.

The Laplace transform procedure used in the previous section cannot be utilized here to solve (8) since it is not readily possible to evaluate

$$
\int_{0}^{\infty} e^{-p t} \int_{0}^{t} B(t-x) \Phi(x, t) d x d t .
$$


Thus with a time dependent net maternity function the convolution theorem (3) does not in general hold. Cerone and Keane [2], [3] were, however, able to evaluate (10) where the net maternity function changed exponentially with time. With

$$
\Phi(x, t)=\left[1 / R+(1-1 / R) e^{-\lambda t}\right] \phi(x), \text { where } \lambda>0,
$$

(10) can be evaluated giving, from (8) using (3),

$$
B^{*}(p)=F^{*}(p)+(1 / R) \phi^{*}(p) B^{*}(p)+(1-1 / R) \phi^{*}(p+\lambda) B^{*}(p+\lambda) .
$$

Cerone and Keane [2] presented a fast converging numerical algorithm for determining the asymptotic value through equations of the form (11).

However, if $\Phi(x, t)$ varies explicitly with time only for $t<x$ (for the parent population) then the convolution is not disrupted and we can readily isolate $B^{*}(p)$. This type of differential maternity behaviour between the parent and subsequent population was mentioned in Keyfitz [7] and later analysed by Frauenthal [4] who allowed the parent population to continue with the old regime while those born after the origin $t=0$ adopted replacement age-specific fertility rates brought about by scaling that of the initial population by the net reproductive rate $R$. He was successful in obtaining the asymptotic behaviour of the ensuing population.

The present model may be looked upon as a generalisation of that presented by Frauenthal [4] allowing for a gradual change in the parent population's reproductive behaviour. Such gradual changes have been acknowledged by a number of authors as being more realistic that the abrupt models (Frejka [5], Keyfitz [8] and Ruzicka [13]).

For simplicity we assume that the population is initially stable such that $B(t)=Q e^{r t}$ for $t<0$. Thus if

$$
\Phi(x, t)= \begin{cases}\phi(x) & \text { for } t<0 \\ \Phi_{1}(x, t) & \text { for } t<x \\ \phi_{2}(x) & \text { for } t>x\end{cases}
$$

equations (8) and (9) give

$$
B(t)=Q e^{r t} \int_{t}^{\infty} e^{-r x} \Phi_{1}(x, t) d x+\int_{0}^{t} B(t-x) \phi_{2}(x) d x .
$$

Consequently we can now take Laplace transforms of (13) unhindered, since the convolution theorem still holds, giving a transform similar to (4),

$$
B^{*}(p)=F^{*}(p) /\left(1-\phi_{2}^{*}(p)\right),
$$


where

$$
F^{*}(p)=Q \int_{0}^{\infty} e^{-(p-r) t} \int_{t}^{\infty} e^{-r x} \Phi_{1}(x, t) d x d t
$$

Following the procedure of the previous section we obtain

$$
B(t) \sim Q_{2} e^{r_{2} t}
$$

where,

$$
\left.\begin{array}{l}
r_{2} \text { is the real root of } \phi_{2}^{*}(p)=1, \\
Q_{2}=F^{*}\left(r_{2}\right) / \kappa_{2}, \\
F^{*}\left(r_{2}\right) \text { is given by }(15), \text { and } \\
\kappa_{2}=\int_{0}^{\infty} e^{-r_{2} x} \phi_{2}(x) d x
\end{array}\right\}
$$

4. Time dependence no greater than the lowest age of childbearing, $\alpha$

A very important case of change in the net maternity function, as described by (12), presents itself if we take into consideration the very nature of the function. In a realistic situation the net maternity function is positive for age $x$ such that $\alpha<x<\beta$ and zero elsewhere. Thus if the net maternity function changes explicitly with time over an interval less than or equal to the lowest age of childbearing $\alpha$ then the convolution is not disrupted.

Consider the effects of a general time dependent scaling of the net maternity function for $\tau \leqslant \alpha$ so that

$$
\Phi(x, t)= \begin{cases}\chi(t) \phi(x) & \text { for } 0<t<\tau, \\ \phi_{2}(x) & \text { for } t>\tau .\end{cases}
$$

Here $\phi(x)$ and $\phi_{2}(x)$ are both positive for $\alpha<x<\beta$ and zero elsewhere. The asymptotic total birth rate is given by (16)-(17) where from (15) and, using (12), (13) and (18)

$$
\begin{aligned}
\kappa_{2} Q_{2} / Q= & F^{*}\left(r_{2}\right) / Q=\int_{0}^{\tau} e^{-\left(r_{2}-r\right) t} \chi(t) d t+\int_{\tau}^{\alpha} e^{-\left(r_{2}-r\right) t} \int_{0}^{\beta} e^{-r x} \phi_{2}(x) d x d t \\
& +\int_{\alpha}^{\beta} e^{-\left(r_{2}-r\right) t} \int_{t}^{\beta} e^{-r x} \phi_{2}(x) d x d t \\
= & \int_{0}^{\tau} e^{-\left(r_{2}-r\right) t} \chi(t) d t+\left(\phi_{2}^{*}(r) e^{-\left(r_{2}-r\right) \tau}-1\right) /\left(r_{2}-r\right)
\end{aligned}
$$

for $\tau \leqslant \alpha$. 
If

$$
\phi_{2}(x)=\phi(x) / R,
$$

then the population tends towards a stationary state $\left(r_{2}=0\right)$ and the total birth rate from (16) and (17) is

$$
B(t) \sim Q_{2}=Q \kappa^{-1} \int_{0}^{\tau} e^{r t} \chi(t) d t+Q\left(R-e^{r \tau}\right) /(r R \kappa),
$$

where $\kappa=R^{-1} \int_{\alpha}^{\beta} x \phi(x) d x$ is the average age of childbearing in the eventual stationary population.

Before considering a number of examples of $\chi(t)$ for which (21) can be evaluated analytically, some observations can be made for a general non-negative function $\chi(t)$.

We note that if $\tau \rightarrow 0$ in (18) with (20) then we obtain the abrupt change to replacement level fertility model of Keyfitz [7] and hence from (21) we correctly obtain the asymptotic value found by Keyfitz, namely,

$$
B(t) \sim Q(R-1) /(r R \kappa) \quad \text { for } r>0 .
$$

The difference $D$ of (22) from (21) gives a comparison between the asymptotic total birth rate resulting from an abrupt Keyfitz change to replacement level fertility with that from a gradual change depicted by (18) and (20), where

$$
D=Q \kappa^{-1} \int_{0}^{\tau} e^{r t} \chi(t) d t+Q\left(1-e^{r \tau}\right) /(r R \kappa) .
$$

It can be stated that for all $t$ in the range $0<t<\tau$, if $\chi(t) \geqslant R^{-1}$ then $D \geqslant 0$, and if $0<\chi(t) \leqslant R^{-1}$ then $D \leqslant 0$. However if $\chi(t)$ takes on values on either side of $R^{-1}$ then it is not obvious whether (21) is greater or less than the asymptotic value, (22), obtained by Keyfitz.

\section{Examples of $\chi(t)$}

$$
\chi(t)=c, \text { a constant. }
$$

With this $\chi(t)$, equation (18) with (20) represents a general step change at the origin. Further, if $\chi(t)=c \neq 1 / R$ then there is also a step change at $t=\tau$. From (21), the asymptotic total birth rate is given by

$$
B(t) \sim Q\left[e^{r \tau}(R c-1)+R(1-c)\right] /(r R \kappa) .
$$

If $c=1 / R$ then

$$
B(t) \sim Q(R-1) /(r R \kappa),
$$

which is equivalent to letting $\tau \rightarrow 0$ thus obtaining the abrupt change to replacement level fertility model of Keyfitz.

If $c=1$ then

$$
B(t) \sim Q(R-1) e^{r \tau} /(r R \kappa)
$$


This model was also mentioned by Keyfitz [7] and represents an abrupt change to replacement at time $t=\tau$, and, not necessarily at the origin.

$$
\chi(t)=\left\{\begin{array}{l}
1-\theta t \\
e^{-\lambda t}
\end{array}\right.
$$

where for continuity $\theta=(1-1 / R) \tau^{-1}$ and $e^{\lambda \tau}=R$.

With this time dependence we obtain, from (21),

$$
B(t) \sim Q \frac{R-1}{r R \kappa} \frac{e^{r \tau}-1}{r \tau}
$$

and

$$
B(t) \sim Q \frac{R-e^{r \tau}}{r R \kappa} \frac{\lambda}{\lambda-r} \quad \text { where } \lambda=\tau^{-1} \ln R,
$$

respectively. Because of the difference in the rate of change of the abrupt, linear and exponential models over the same length of time $\tau$, the resulting asymptotic values (25), (26a) and (26b) are in order of decreasing magnitude.

It is of interest to note that a given asymptotic value may possibly be obtained as a result of more than one particular type of time dependence. For example, given a linear change to bare replacement for $0<t<\tilde{\tau}$ then an abrupt change at

$$
\tau=r^{-1} \ln \left(\left(e^{r \tilde{\tau}}-1\right) /(r \tilde{\tau})\right),
$$

obtained from equating (25) with (26a), will produce the same asymptotic total birth rate. Care must be taken with such problems, however, since we have the restriction on $\tau$ that $0<\tau \leqslant \alpha$, and no such $\tau$ may exist.

The converse problem for an abrupt, linear or exponential change on $0<t<\tau$ $\leqslant \alpha$ can also be solved. Thus, given an asymptotic total birth rate $Q_{2}$, we can determine $\tau$ (which characterises the particular time dependence $\chi(t)$ ) from (25), (26a) and (26b) depending on whether an abrupt, linear or exponential change is assumed. Some root finding procedure would have to be used to find $\tau$ from (26). However $\tau$ can be found directly from (26) in the case of an assumed abrupt change. No such $\tau$ will exist if the given asymptotic value is outside the interval between the upper and the lower bound which occur, for $\chi(t)$ monotonically decreasing, at $\tau=\alpha$ and $\tau=0$ respectively. The converse problem is thought to have possible population management implications.

$$
\chi(t)=\sum_{n=1}^{N} \sigma_{n} H\left(t-t_{n-1}\right) H\left(t_{n}-t\right)
$$

where $t_{0}=0, t_{N}=\tau \leqslant \alpha$, and $H$ is the Heaviside unit function. 
This time dependence represents a histogram and thus allows great flexibility in the type of change involved. It allows for variable width $\left\{h_{n}\right\}$ of the rectangles, where

$$
h_{n}=t_{n}-t_{n-1}, \quad n=1,2, \ldots, N,
$$

and thus wider rectangles may be used where the change is gradual, while narrower rectangles are used when the change with time is rapid. The histogram approximation may be used when the change is expected to be so irregular that a simple mathematical curve is unable to represent it.

From (21), $B(t) \sim Q_{2}$ where, with the histogram $\chi(t)$,

$$
Q_{2}=\frac{Q}{r \kappa} \sum_{n=0}^{N} \tilde{\sigma}_{n} e^{r t_{n}}+\frac{Q}{r R \kappa}\left[R-e^{r \tau}\right],
$$

with

$$
\begin{aligned}
& \tilde{\sigma}_{0}=-\sigma_{1} \\
& \tilde{\sigma}_{n}=\sigma_{n}-\sigma_{n+1}, \quad \text { for } n=1,2, \ldots, N-1, \text { and } \\
& \tilde{\sigma}_{N}=\sigma_{N} .
\end{aligned}
$$

An example of $\chi(t)$, for which (21) can be evaluated analytically, which is more of theoretical interest because of its complexity compared with the relative lack of knowledge of the change, is provided by

$$
\chi(t)=\sum_{n=0}^{N} P_{k_{n}}(t) e^{-b_{n} t}
$$

where $P_{k_{n}}(t)$ is a polynomial of order $k_{n}$. With this $\chi(t)$ in (21), integrals of the form

$$
I_{n}=\int_{0}^{\tau} t^{n} e^{-b t} d t
$$

result, which can be evaluated by integrating by parts to obtain the following recurrence relation

$$
\begin{aligned}
& I_{n}=\frac{-1}{b}\left[t^{n} e^{-b t}\right]_{0}^{\tau}+\frac{n}{b} I_{n-1} \quad \text { for } n=1,2, \ldots, \\
& I_{0}=\left(1-e^{-b \tau}\right) / b
\end{aligned}
$$

\section{Time dependence defined in a piecewise fashion}

Frauenthal's model on differential fertility between the parent and the subsequent populations can be generalized to include time dependent changes in the net maternity function. As stated earlier in Section 2, the Laplace transform 
method is not hampered if the net maternity function changes explicitly with time for the parent $(t<x)$ population. It has been shown however, that exponential time dependence can be accommodated using the methods developed and analysed in Cerone and Keane [2], [3]. Thus we may have a time dependent net maternity function of the form

$$
\Phi(x, t)=\left\{\begin{array}{l}
\Phi_{1}(x, t) \quad \text { for } t<x, \\
\Phi_{2}(x)+e^{-\lambda(t-x)}\left[\phi_{1}(x)-\phi_{2}(x)\right] \quad \text { for } t>x,
\end{array}\right.
$$

where $\lambda>0$, and, for continuity at $t=x$, we assume $\phi_{1}(x)=\Phi_{1}(x, t)$. Frauenthal's model was not continuous.

If the population is initially stable we thus have the total birth rate being given by

$$
\begin{aligned}
B(t)= & Q e^{r t} \int_{t}^{\infty} e^{-r x} \Phi_{1}(x, t) d x \\
& +\int_{0}^{x} B(t-x)\left[\phi_{2}(x)+e^{-\lambda(t-x)}\left[\phi_{1}(x)-\phi_{2}(x)\right]\right] d x .
\end{aligned}
$$

To obtain the asymptotic behaviour, we proceed in a formal fashion by using Laplace transform techniques outlined in Section 2. The Laplace transform of (29) yields

$$
\left[1-\phi_{2}^{*}(p)\right] B^{*}(p)=F^{*}(p)+B^{*}(p+\lambda)\left[\phi_{1}^{*}(p)-\phi_{2}^{*}(p)\right],
$$

with $F^{*}(p)$ being given by (15).

Without loss of generality, we can assume that $\phi_{2}^{*}(0)=1$ so that the population eventually becomes stationary, that is, $B(t) \sim Q_{2}$ where on letting $p \rightarrow 0$ in (30) we have

$$
\kappa_{2} Q_{2}=F^{*}(0)+B^{*}(\lambda)\left[\phi_{1}^{*}(0)-1\right],
$$

with

$$
\kappa_{2}=\int_{0}^{\infty} x \phi_{2}(x) d x .
$$

Now to evaluate $Q_{2}$ from (31) we need to know $B^{*}(\lambda)$ which can be obtained from (30) by setting up a recurrence relation similar to the method of Cerone and Keane [2], [3]. Thus, putting $p=n \lambda$ in (30) gives

$$
B^{*}(n \lambda)=\delta_{n}+\varepsilon_{n} B^{*}((n+1) \lambda) \text {, }
$$

where

$$
\delta_{n}=\frac{F^{*}(n \lambda)}{1-\phi_{2}^{*}(n \lambda)}
$$


and

$$
\varepsilon_{n}=\frac{\phi_{1}^{*}(n \lambda)-\phi_{2}^{*}(n \lambda)}{1-\phi_{2}^{*}(n \lambda)} .
$$

Successive approximations to $B^{*}(\lambda)$, and hence from (31) to $Q_{2}$, are obtained by assuming $B^{*}(N \lambda)=0$ for some positive integer $N$, in (32).

A time dependent change in the net maternity function to replacement level fertility over $0<t<\tau \leqslant \alpha$ which was presented in Section 4, represents in many circumstances an unachievable goal. At best, the change allowed is up to $t=\alpha$ $(=10$ or 15 years $)$ whereas a number of authors have indicated a longer period of time for the change to replacement fertility behaviour. Frejka [5] allows for a linear change over $0,10,30,50,70$ years of the Gross Reproduction Rate and thus presents a range of alternatives that would be meaningful for both the more and the less developed countries. Keyfitz [7] states that a gradual drop over 30 years would be more likely.

Although it is difficult to anticipate the effects of certain policies which would produce changes in the fertility behaviour of a population, one can imagine that such a change would vary most in the short term before settling down. We now introduce a model which allows for a general time dependent change over $0<t<\tau \leqslant \alpha$ with a simpler (exponential) change for $t>\alpha$.

Thus we consider the time dependent net maternity function to be given by

$$
\phi(x, t)=\left\{\begin{array}{l}
\chi(t) \phi(x) \quad \text { for } 0<t<\tau<\alpha, \\
{\left[1 / R+\left(a_{\tau}-1 / R\right) e^{-\lambda(t-\tau)}\right] \phi(x) \quad \text { for } t>\tau,}
\end{array}\right.
$$

where

$$
\begin{gathered}
\chi(0)=a_{0}, \quad \chi(\tau)=a_{\tau}, \quad \lambda>0 \\
\phi(x)>0 \text { for } \alpha<x<\beta \text { and } \phi(x)=0 \text { elsewhere. }
\end{gathered}
$$

Now,

$$
B(t)=Q e^{r t} \int_{t}^{\beta} e^{-r x} \Phi(x, t) d x+\int_{\alpha}^{t} B(t-x) \Phi(x, t) d x,
$$

and hence using (33) we get, on taking Laplace transforms,

$$
\left[1-\phi^{*}(p) / R\right] B^{*}(p)=F^{*}(p)+\left(a_{\tau}-1 / R\right) e^{\lambda \tau} \phi^{*}(p+\lambda) B^{*}(p+\lambda),
$$

where

$$
\begin{aligned}
F^{*}(p) / Q= & \int_{0}^{\tau} e^{-(p-r) t} \chi(t) d t+\int_{\tau}^{\alpha} e^{-(p-r) t}\left[1 / R+\left(a_{\tau}-1 / R\right) e^{-\lambda(t-\tau)}\right] d t \\
& +\int_{\alpha}^{\beta} e^{-(p-r) t}\left[1 / R+\left(a_{\tau}-1 / R\right) e^{-\lambda(t-\tau)}\right] \int_{t}^{\beta} e^{-r x} \phi(x) d x d t .
\end{aligned}
$$


Hence on, simplifying,

$$
F^{*}(p) / Q=\int_{0}^{\tau} e^{-(p-r) t} \chi(t) d t+f(p) / R+\left(a_{\tau}-1 / R\right) e^{\lambda \tau} f(p+\lambda)
$$

where

$$
f(p)=\left(e^{-(p-r) \tau}-\phi^{*}(p)\right) /(p-r) .
$$

The asymptotic birth rate $Q_{2}$ is obtained from (34) by letting $p \rightarrow 0$ to give

$$
\kappa Q_{2}=F^{*}(0)+\left(a_{\tau}-1 / R\right) e^{\lambda \tau} \phi^{*}(\lambda) B^{*}(\lambda),
$$

where the methods of Cerone and Keane [2], [3] as outlined earlier in this section would have to be utilized to obtain approximations to $B^{*}(\lambda)$ by setting up a recurrence relation from (34).

\section{Discussion}

The methods for obtaining the asymptotic behaviour of a population have been extended to treat a piecewise time dependent net maternity function. It has been shown that the convolution theorem is still applicable if the net maternity function has no time dependence associated with ages greater than the minimum age of childbearing and less than the current time.

It has also been shown that time dependence associated with ages less than the current time may be described if that time dependence is exponential. These extensions allow for a great variety of paths of change in the net maternity function to obtain the asymptotic behaviour of the population which until now have not been possible.

\section{References}

[1] R. Bellman and K. L. Cooke, Differential difference equations, (Academic Press, New York, 1963).

[2] P. Cerone and A. Keane, "The momentum of population growth with time dependent net maternity function", Demography 15 (1978), 131-134.

[3] P. Cerone and A. Keane, "The stable births resulting from a time dependent change between two net maternity functions", Demography 15 (1978), 135-137.

[4] J. C. Frauenthal, "Birth trajectory under changing fertility conditions", Demography 12 (1975), 447-454.

[5] T. Frejka, The future of population growth: alternative paths to equilibrium (Wiley, New York, 1973).

[6] N. Keyfitz, Introduction to the mathematics of population, (Addison-Wesley, Reading, Mass., 1968). 
[7] N. Keyfitz, "On the momentum of population growth", Demography 8 (1971), 71-80.

[8] N. Keyfitz, "Reproductive value: with applications to migration, contraception and zero population growth", in Quantitative sociology: international perspectives on mathematical and statistical modelling. H. M. Blalock et al. (Eds.), (Academic Press, New York, 1975), 587-612.

[9] N. Keyfitz, Applied mathematical demography, (Wiley, New York, 1977).

[10] A. Lopez, Problems in stable population theory (Office of Population Research, Princeton, 1961).

[11] S. Mitra, "Influence of instantaneous fertility decline to replacement level on population growth", Demography 13 (1976), 513-519.

[12] J. H. Pollard, Mathematical models for the growth of human populations (Cambridge University Press, 1973).

[13] L. T. Ruzicka, Reflections on zero growth of the Australian population, National Population Inquiry-Research Report No. 7 (Canberra: Australian Government Publishing Service, 1977).

[14] F. R. Sharpe and A. J. Lotka, "A problem in age distribution", Philosophical Magazine 21 (1911), 435-438.

[15] K. P. Tognetti, "Some extensions of the Keyfitz momentum relationship", Demography 13 (1976), 507-512.

Department of Mathematics

The University of Wollongong

Box 1144

Wollongong

N.S.W. 2500 